\title{
Symbolically Mediated Interaction and Perspective- taking: A Social-relational Perspective on Social Cognitive Development
}

\author{
Duygu Uygun Tunc \\ University of Heidelberg \\ duygu.uygun@outlook.com
}

Received 15 August 2018; accepted 17 May 2019; published 31 December 2019

\begin{abstract}
It is widely agreed that perspective-taking plays an important role in the development of children's understanding of themselves and others as social agents with their own beliefs, desires, goals, and representations of the world. However, how perspective-taking is realized and how the ability of perspective-taking develops is a matter of dissensus. The two theories currently dominating social cognition research, theory-theory, and simulation-theory construe perspective-taking as modeling, thus as an individual and inferential process. Interactionist theories prioritize interpersonal interaction but deny perspective-taking a constitutive role by arguing for a basic, immediate understanding of self and others in interaction. Cognitivist accounts downplay the role of interaction, while interactionist accounts overemphasize the role of subsymbolical processes. What is central to perspective-taking and its development, but missing in either approach is symbolically mediated interaction. The social-relational perspective dating back to Lev Vygotsky and George Herbert Mead cuts across this schism and offers valuable insight into how perspective-taking develops through symbolic activity within a social context. Adopting the basic elements of the social-relational framework, the present work argues that understanding of self and others depends on the development of perspective-taking ability through symbolically mediated interaction. Perspectives are primarily differentiated, assumed, and coordinated within social interaction and subsequently through the individual, cognitive operation of perspective-taking. Symbolic mediation facilitates this transition from the social enaction of perspective-taking to mental construal and coordination of perspectives by transforming the structure of action. Higher order mental processes are not presupposed but constituted by social interaction through the child's internalization of the perspectival structure of symbolic communication.
\end{abstract}

Keywords: perspective-taking; symbolic interaction; cognitive development; social interaction; cognition 


\section{Introduction}

For the bigger part of the last century, perspective-taking was arguably the most frequently used term in the literature on social cognition. In recent decades, it handed over its prevalence to terms such as theory-of-mind, mindreading, mentalizing, or folk psychology as social cognition increasingly began to be seen as a matter of mental-state attribution. Nonetheless, contemporary researchers often use these terms interchangeably with perspective-taking or still feature perspective-taking in their theoretical explanations of mental-state attribution, commonly specifying it with adjectives such as visual/perceptual (e.g., Flavell, 1992), conceptual (e.g., Shantz, 1983; Clark, 1997; Tomasello, 1999), epistemic (e.g., Perner, 1991) or affective/emotional (e.g., Vaish, Carpenter, \& Tomasello, 2009).

In parallel to the changes in terminology, how the nature and developmental role of perspective-taking are construed across the century shows significant differences in theoretical orientation (Carpendale \& Lewis, 2006). Contemporary theorizing on social cognition differs from earlier developmental theories such as those of Baldwin (1894/1906), Mead (1934), Piaget (e.g., 1928, 1945/1962, 1974/1977), and Vygotsky $(1930 / 1978,1934 / 1987)$ in its relative disregard for social interaction. For earlier psychologists, perspectives are differentiated and coordinated primarily in interactive contexts, as positions or roles, and subsequently through meta-representational processes. In contemporary theorizing, however, meta-representational processes are primary and enabling for social understanding in interaction. Accordingly, perspective-taking is restricted to an individual cognitive modelling process aimed at explaining or predicting others' behavior by attributing beliefs, intentions, or feelings.

In response to the over-mentalistic orientation of the major social cognition theories such as theory-theory and simulation theory, and as an extension of the embodied-enactive approach to cognition, interaction theory has recently been proposed as the latest addition to the theoretical explanations of social cognition. This proposal argues against the mainstream "mentalstate attribution" conception and posits immediate, embodied social interaction as being constitutive of social cognition. Interaction theory, however, constitutes another radical departure from the earlier theories in that it puts reflective perspective-taking, which it equates with meta-representational modelling, completely out of the picture. There emerges, as a result, a theoretical gap between embodied, immediate and mediated, reflective forms of intersubjectivity. What is central to perspective-taking and its development but missing in either approach, we argue, is symbolically mediated interaction.

The aim of this paper is two-fold: to put research on social cognitive development into perspective, both historically and theoretically, and to present the main outlines of a social-relational approach that is inspired largely by the developmental theories of Lev Vygotsky and George Herbert Mead, and in part by the Piagetian perspective-taking tradition. We think that the social-relational perspective harbors several important insights of both contemporary metarepresentational and interactionist accounts without dichotomizing reflective and pre-reflective, mediated and immediate forms of social cognition. The key to bridging the unnecessarily divided picture of the mind is changing the order of explanation endorsed in meta-representational theories; namely, positing social interaction as explanans and individual, higher order cognitive processes as explanandum. 
First, we argue that perspective-taking is primarily social-relational and secondarily individual and cognitive. Perspectives are first differentiated, assumed, and coordinated within social interaction and through pragmatic involvement with a socially mediated environment, and later the social operation is internalized and transformed into the cognitive ability of perspectivetaking. Secondly, the development of the capacity for perspective-taking is essentially related to the development of the capacity for sign-use. We conclude that through the course of development it is through social interaction that higher order sign-relations, or symbolic activity come about, which in turn opens up further levels of social meaning and hence deepens and enriches intersubjective processes.

\section{The Piagetian perspective-taking tradition and the theory-of-mind approach}

Research on children's social cognitive capabilities largely began, as it is the case in many other areas of cognitive development, with Piaget, who conceived social cognitive development as a development of perspective-taking skills (Flavell, 2000; Flavell \& Miller, 1998). Within the Piagetian framework, social cognitive development involves the child's coming to know that there are perceptual, affective, and conceptual perspectives through ongoing interactions with others, and becoming gradually more skillful in taking and coordinating different perspectives (Piaget, 1928, 1977; Piaget \& Inhelder, 1963; Shantz, 1983). What underlies this development is the transformation of the child's egocentric relation to the world through a process of decentration: the child gradually understands that his or her immediate relation to the world is not the only one, but a perspective among those of others. In cognitive terms, the child becomes able to entertain and coordinate multiple, non-exclusive perspectives on an object, event or situation on a representational level and eventually comes to understand the notion of perspectives. The end-point of cognitive development, accordingly, is the ability to have second-order thoughts; in other words, to take a higher-order perspective on one's own personal perspective. Put in terms of a transformation of the child's relation to the world, social cognitive development is closely linked to the whole range of changes in representational capabilities that are central to moral development, scientific thinking and verbal communication. ${ }^{1}$

Since the 80s, research in the field focuses mainly on what is called a "theory-of-mind." Originally coined in the framework of primate cognition (Premack \& Woodruff, 1978), theory-ofmind soon entered the theoretical toolset of developmental psychologists. In the introduction to the first book on cognitive development to feature this term, it is defined as a set of interconnected concepts for representing mental states as representations, or "a set of meta-representations" (Astington, Harris, \& Olson, 1988). Having a theory-of-mind implies the ability to attribute mental states to others and oneself, and to understand that mental representations might differ from person to person and from reality, which the extensive body of research through false-belief attribution (Wimmer \& Perner, 1983), appearance-reality paradigms (Flavell, Flavell, \& Green, 1983) addresses. While the perspective-taking tradition construes social understanding in terms of differentiating another person's perspective from one's own, the the-

\footnotetext{
${ }^{1}$ For a review, see Flavell (1992).
} 
ory-of-mind approach articulates it in terms of attributing to others' beliefs, desires or intentions that may be different than one's own in reference to the same situation. Cognitive development culminates in both approaches in the ability to have higher-order representations of others' or own representations of the world, understood as such. Thus, the perspective-taking and the theory-of-mind frameworks address similar competencies and have considerable theoretical overlaps. However, while the former conceives this end in terms of attaining a global coordination of perspectives and acquiring the ability to continuously situate oneself within and with respect to it, the latter does so in terms of acquiring a representational theory-ofmind.

Older researchers or those few who have worked in both frameworks such as Flavell (1992) emphasize the continuity of these approaches, although there are considerable differences in particular theoretical explanations. The most important aspect of continuity for the purposes of this paper is the appeal to a higher-order representational picture of social cognition, which is also linked to self-knowledge along similar mechanisms. The most important aspects of divergence are related to the different construals of the origin of higher order mental processes.

The general guiding principle of Piaget's account of origins is that higher functions develop out of lower regulatory functions as the child continuously tries to adapt its actions to the challenges of the environment. Development is a transformation in a direction that goes from action to thought. Cognitive processes originate accordingly in the coordinative structure of actions (Mounoud, 1996). This general theoretical orientation is found also in the accounts of Vygotsky and Mead, although their emphasis is on interaction instead of individual action, and on the sociocultural properties of the environment that support mental development. In Piaget's theory, the role of social interaction in cognitive development is confined to introducing cognitive conflict and thereby forcing children to make attempts individually at reconciling different points of view and achieve equilibrium (Rogoff, 1999, p. 72). At the opposite end are the innatist accounts that argue for a theory-of-mind "module" (e.g., Baron-Cohen \& Ring, 1994). Modular accounts risk begging the question of mind for reasons we will go into in the context of the theories of Mead and Vygotsky. In the following we will instead go into theories that assign experience a foundational, formative role; namely, theory-theory and simulation theory.

\section{Theory, simulation or embodied interaction}

The majority of philosophical accounts of social cognition fall into either third-person inference-based theory-theory (e.g., Carruthers, 2009; Gopnik \& Meltzoff, 1997; Perner, 1991) or first-person inference-based simulation theory (e.g., Gallese \& Goldman, 1998; Goldman, 2006; Gordon, 1986). According to theory-theory, interpersonal understanding depends on our general knowledge of minds, which derives from a foundational folk psychological theory formulated and sophisticated throughout development. This theory comprises conceptions of what the most basic elements of the mind are, such as desires and beliefs, and how these are related to one another, perceptions, and behaviors (Flavell, 2000). It enables us to conceive others as well as ourselves as minded agents and is used to infer causes and trajectories of actions. Moreover, failure in mental-state attribution is due to deficits in folk psychological 
theory, such as applying a desire-psychology as opposed to a belief-desire psychology (Bartsch \& Wellman, 1995). Perspective-taking in the theory-theory context arguably amounts to building a theoretical model of another person's perspective based on what we know about mental states in general and about his or her mental states in particular. It is reasonable to think that the ability to take and coordinate perspectives has a knowledge aspect as well as process aspect. Similar to the distinction between metacognitive knowledge and metacognitive monitoring (Flavell, 1979), we can speak of a general knowledge of the perspectivity of perceptions, beliefs, attitudes, or desires, as well as processes of taking and coordinating personal perspectives. Consequently, it is hard to reject that some theoretical element is involved in social cognition, although explanations of how this theory is established might differ. The "child scientist" theory proposes that children formulate, test, and revise their theories in the light of accumulating evidence in a fashion similar to scientific hypothesis testing and theory revision (Scholl \& Leslie, 1999). This perspective arguably overlooks, however, how formative culturally mediated social interaction patterns, child-rearing practices, social institutions, and cultural artifacts can be in the formulation of such a theory. Besides the difficulty of isolating sociocultural factors from actual scientific practices, it is close to impossible to observe or theoretically construct what the individual, solitary efforts of the child in theory-building would yield.

Simulation theory criticizes theory-theory for painting an over-intellectualized picture of social cognition (Gordon, 1986). It argues, instead, that we already have our own experiences on the basis of which we can simulate and understand other minds. Simulation theory places the emphasis on the process aspect of perspective-taking and argues that social understanding consists in the mental simulation of the perspective of another person inside one's own, experiencer's or first-person perspective instead of relying on theoretical inferences from a thirdperson or observer's perspective. Simulation is realized by creating pretend states through imagination, running these through a suitable cognitive mechanism and finally attributing the outputs to the other person (Shanton \& Goldman, 2010). This higher-level, imagination-based simulation, on the other hand, is not the only mechanism for mindreading. Low-level mindreading can take place through sub-personal, embodied mirroring mechanisms. Self-understanding can also be explained in simulation terms: we project ourselves into the past and the future through episodic memory and prospection, or mental time-travel (Shanton \& Goldman, 2010).

It is reasonable to argue that the close association between the terms first-person and thirdperson perspective and the subjective and the objective, or the experiencing and observing perspectives, on the other hand, presents serious difficulties in the context of social interaction, because it limits the sphere of subjectivity to the "inside" of a person and renders the "outside" bereft of subjectivity, leading to the problems of either how to recognize the other as another subject whose experience is as real as one's own, or how to go beyond one's own limited experience and actually take the perspective of the other. These two "main roads" converge on the point that social cognition is a matter of making inferences of others' mental states-a special application of a general epistemic procedure - and social interaction is not a fundamental category for understanding how we assume, change, and coordinate social perspectives, which is at the end of the day an individual cognitive activity. 
In order to acknowledge the phenomenon of interaction as introducing new conditions of its own which are not analyzable into the subjective experience and objective observations of both parties taken separately, there is increasing interest in the notion of second-person perspective, which is taken to be the perspective of interaction, through interesting connections between phenomenology, neuroscience, developmental psychology and systemic approaches (e.g., De Jaegher, Di Paolo, \& Gallagher, 2010; Gallagher, 2001; Gallese, 2014; De Jaegher \& Di Paolo, 2007; Reddy \& Morris, 2004).

This third path is opened up upon making a very important point regarding an apparent dualistic impasse inherent in the field: how successfully can we understand intersubjectivity if we dissolve it into objective or subjective processes and exclude the prefix "inter" from the discussion? This group of theories, going back to Gallagher's work (2001), which are commonly labelled as the interaction theory reject the mindreading or mental-state attribution picture of intersubjectivity in favor of a shared, second-person meaning-making account. Interactionists criticize theory-theory and simulation theory on the grounds that both offer representationheavy, non-interactional accounts of social cognition and argue instead for an immediate, enactive and embodied understanding of self and other in social interaction through non-reflective processes such as imitation, gaze following, or social referencing. Directly visible gestures, expressions and movements constitute the backbone of embodied and enactive social understanding. They argue, moreover, that embodied, enactive engagement with others is not only the developmentally primary but also the default mode of adult intersubjectivity (Gallagher, 2001; De Jaegher et al., 2010).

Interaction theory presents a seemingly eclectic synthesis of phenomenology, dynamic systems theory, and rhetoric. This synthesis is brought about thanks to the common anti-representationalist ground which translates into a downplaying of higher order cognitive processes, and symbolic thinking in particular, in favor of pre-reflective experience and embodied intersubjectivity. The central ideas of the interaction theory can be specified as those of direct perception (Gallagher 2008), attunement or mutual incorporation (Fuchs \& De Jaegher, 2009; De Jaegher et al., 2010), participatory sense-making (De Jaegher \& Di Paolo, 2007), and narrative practices (Gallagher \& Hutto, 2008).

The notion of direct perception has a phenomenological origin and a relatively longer history to it, the notions such as (affective) attunement and mutual incorporation (a process in which the lived bodies of both participants extend and form a common intercorporality) deal with a phenomenology of embodied interaction - so can be seen as having both phenomenological and dynamic systems aspects. Participatory sense-making is a notion developed directly from the dynamic systems point of view and emphasizes interaction and coordination of agents. This group of notions have in common that they are employed to describe experience and interaction only synchronically and only pre-symbolically. Thus, historicity of meaning as well as its social and cultural aspect are necessarily missing, hence any more sophisticated meaning-making activity directed at one's own experience or at the actions of others escapes their scope. The direct perception view takes meaning for granted. Dynamic systems theoretical accounts, on the other hand, tend to explain meaning-making always as an emergent process. In order to make up for this insufficiency without endorsing representationalist elements such as inference or projective attribution, interaction theory is lately supplemented with the 
concept of narration. The notion of narrative practice is considered to complement synchronic, embodied, interactive meaning-making with narratives that are ubiquitous in any culture and allow us to implicitly frame the actions of others and our past experiences. Since narratives can be symbolically articulated, they are considered to enable intelligible and communicable understanding of experience and behavior. On the other hand, de Bruin and de Haan (2009) argue that the notion of narrative practice is an eclectic and inadequate addition to the theoretical palette, because its formulations either fail to cover theory-driven aspects of social cognition or involve implicit folk psychological elements incompatible with enactivism.

We think that the starting point of the second-person approach is not only very legitimate, but also answers to a significant lacuna in the field of social cognition. Moreover, enactivism shares certain fundamental tenets such as the developmental priority of action, interaction and shared practices of meaning-making with the social-relational approach, although without a historical connection. But then again, various research programs travelling this third path are united around a common rejection of "mentalistic" categories such as representation in the explanation of cognitive processes in favor of more rudimentary experiential, co-ordinational and bodily phenomena. What is common to the perspective-taking tradition and the theory-ofmind approach, on the other hand, is the acknowledgement that social-cognitive development requires a gradual transition from dependence on immediate bodily experience in here-andnow and on the concretely present aspects of the context towards more context-independent, mediated, reflective forms of social understanding. Without an adequate and coherent account of reflective, higher order processes and how they might develop out of embodied interactions, the interactionist proposal is not explaining social cognition but explaining it away. Interactionists in fact acknowledge the significance of this problem. De Jaegher and Froese (2009, p. 439) refer to it as the "cognitive gap," and state that the biggest challenge is to account for the upper floors of social cognition while remaining within the enactivist framework.

Intersubjectivity has as much to do with understanding social situations from one's own and others' attitude towards them reflectively, on the basis of social, cultural, and historical meanings that constrain and determine interactive situations, as much as it has to do with attunement, coordination, synchronization, or harmony emerging in interactions. To do away with social representations takes historicity, generalizability, hence symbolic content out of intersubjectivity in favor of bringing mind into the open, lived world of social interaction.

We have, then, at our hands what might in fact be a false dilemma between representation and interaction, between higher order cognitive processes and sub-symbolical processes or "unmediated" processes - as if they are necessarily antithetical. The problem, we argue, would not be solved by downplaying the role of perspective-taking and symbolic mediation, but by shifting the analytic priority from individual mental processes to the meaning-making relation to the world, to others and to oneself and how this fundamental relation acquires more complex and richer forms in development through interpersonal interaction. Enactivism undertakes this reversal of priority by defining cognition as "the enactment of a world and a mind on the basis of a history of the variety of actions that a being in the world performs" (Varela et al., 1991), but so far remains inadequate in explaining the origins of higher order processes. The key elements required for a complete account are already found in the tradition of social-relational theories such as the social act theory of Mead and the sociohistorical psychology of Vygotsky, 
which live on under symbolic interactionism ${ }^{2}$ and sociohistorical activity theory. ${ }^{3}$ According to both schools, symbolic mediation implies sociocultural mediation and cognitive development and it is to be understood within and through sociocultural development (e.g., Bruner, 1990; Cole, 1985; Nelson, 1998; Rogoff, 2003; Wertsch, 1998).

\section{An outline of the overlapping perspectives of Mead and Vygotsky}

Both of these interestingly similar approaches focused on how perspective-taking, and any higher cognitive function for that matter, emerges and develops through symbolic activity within a social context. For both thinkers, any valid theory of the intellectual powers of man and of the emergence of the elaborate socio-cultural artifacts distinguishing his lived-world from his natural habitat were to be provided with an evolutionary and historical account that integrates phylogeny and ontogeny. Man was immersed in ongoing interaction with the environment, through the course of which both were transformed; culture entered the scene of nature as the medium through which this interaction was further deepened and radically altered, by providing a higher stage in the dialectical process. The environment for man was ultimately a social environment, where any — even seemingly direct —interaction with nature was mediated by social experience. Sociality was for Mead and Vygotsky the sole and the key mediator by which higher powers of the mind that render the human animal a self-conscious agent arose, and the social process was fundamental in that it marked a qualitative leap that re-structures human activity with its perceptive, cognitive, and emotional factors, as well as the world in which it takes place (see Côté, 2016). The mind was the product of enculturation.

The nature of higher mental functions could only be understood by analyzing the interactional context within which they develop, following a pattern that goes from intersubjective to intrasubjective, from the outside to the inside, and from practical interactivity to mental activity. For Vygotsky and Mead, the appearance of the cultural world of symbolical communication in evolutionary history indicated a qualitative change in the structure of human behavior. The roots of complex human behavior and any intellectual activity was to be sought in the nature and history of symbolic communication, which enabled both coordination of action and creation of shared meaning. Although a mental activity, symbolic thinking had strong structural affinity with symbolic communication in that speech and thinking became inseparably intertwined activities through the development of sign use.

Although neither Vygotsky nor Mead presented a theory of sign per se of their own, they rather focused on the emergence and development of signification and meaning as integral to the phylogenic and ontogenic development of mind and self. The Vygotskian and the Meadian conceptions of the sign process both deeply resonate with certain central characteristics of Peirce's semiotics such as the historical and social understanding of meaning, the unification

\footnotetext{
${ }^{2}$ The term "symbolic interactionism" was coined by Herbert Blumer (1969), a student of Mead, and among the founders of the tradition Charles Horton Cooley, contemporary and colleague of Mead, and Dewey are also counted.

${ }^{3}$ Cultural-historical activity theory has found wide reception especially since the 1990s, through the works of Michael Cole, James V. Wertsch, and Yrjö Engeström. See e.g., Michael Cole, Yrjö Engeström, and Olga Vasquez (1997).
} 
of thought and communication in the sign process, and the dialogical conception of the sign as the product of a two-way determination by utterance and interpretation. To begin with the last element, Peirce conceives the sign as a welding of two processes, utterance and interpretation, into a unity:

signs require at least two Quasi-minds; a Quasi-utterer and a Quasi-interpreter; and although these two are at one (i.e., are one mind) in the sign itself, they must nevertheless be distinct. In the Sign they are, so to say, welded. Accordingly, it is not merely a fact of human Psychology, but a necessity of Logic, that every logical evolution of thought should be dialogic (CP 4.551). ${ }^{4}$

This dialogic conception of the sign finds reflection in Mead's notion of a "significant symbol," in which two perspectives, that of the self and other, are intertwined. The paradigmatic instance of a significant symbol is the spoken word, which one has to hear and understand at the same time in order to utter. Vygotsky's notion of "word meaning" presents another interesting parallel. For Vygotsky a word meaning is at the same time the embodiment of a concept and the medium for communicating that thought in social interaction, thus a union of thought and speech as well as of representation and communication. Moreover, for Peirce all thought and communication, in the broad senses of cognition and intersubjectivity, is in and through signs; hence any notion of immediate understanding of self or other is misguided. Any potentially conscious cognition is in principle public, because representation to oneself and to another is essentially the same process of semiotic mediation.

In line with the old Platonic conception, Peirce acknowledges that all thought is necessarily a sort of dialogue, "an appeal from the momentary self to the better considered self of the immediate and of the general future" (EP 2.477). Thinking achieves the double determination of utterance and interpretation through its temporal structure and the functional split in the conscious self. A thought is a sign uttered by the ego of a previous moment, addressed to a future thought, effect, or a resolution to act. To the degree that the subject is endowed with memory and imagination, and is proficient in using signs, the thought can switch between utterance and interpretation. What Peirce adds to the Platonic picture is the semiotic and perspectival conception of the self, where the semiotic subject can and does assume the perspective of a community, a conception which will find further elaboration and sophistication in Mead's distinction between "I" and "me". The model case of sign process or semiosis is clearly heterocommunication, where there is an addresser and an audience. The person accomplishes semiosis in thought through becoming its own audience. The paradigm that guides the definitions and classifications of the sign, in parallel, is the external, communicated sign.

Moreover, adherence to a semiotics of production of meaning in diachronic and recursive relationships between the elements of the sign instead of a synchronic theory of signification, as Vygotsky and Mead also do, is precisely what makes a social and historical account of the development of mind and self conceivable.

\footnotetext{
${ }^{4}$ The term quasi-mind has to be understood in the context of Peirce's broader metaphysical argument that thought, or in its more general form "thirdness", is not restricted to the human being but appears in the organic world and develops there. Although this topic is not relevant here, the term has not been taken out in order not to misrepresent Peirce's semiotics as being confined to the human cultural realm.
} 
Mead stands in a close relation to Peirce inasmuch as he focused his theoretical attention on the social nature of knowledge construction and the social formation of the mind. It was Peirce who placed the focus in analyzing action not on personal meaning making, but on the community (Edwards, 2007). For Mead, the key to achieve the unifying goal of the pragmatist program; that is, to overcome the dualism of mind and world was to be looked for neither in the individual construction of knowledge, nor in the individual action within environment, but in society. To be more precise, sign process as the dynamic nexus of mind and world was social and historical, and thereby constituted the most suited unit of inquiry in order to grasp both directions of determination: from the interpersonal to the social and from the social to the individual. Notwithstanding the differences in their sociological and political conceptions of the individual and society, these key points of commonality between Mead and Peirce are also the key points of convergence between the work of Mead and that of Vygotsky.

\section{A semiotic-pragmatic notion of perspective}

We have briefly touched upon various lacunae in the contemporary literature on social cognition that leave differing aspects of the phenomenon of intersubjectivity systematically unaccounted for. To reiterate the previously made points in the form of a hypothesis; intersubjectivity fundamentally involves perspective-taking, which is grounded in actual, situated social interactions that are embedded in a common and meaningful world within which the individuals orient themselves through action and articulate their orientation and that of others symbolically.

In order to substantiate this hypothesis, we need to first re-formulate the notion of perspective(s) in a language that does not dichotomize experience and reflection, situated interaction and general social meanings, and subjectivity and objectivity. Mead's account of perspectivity offers a rich basis for such a formulation, which we will interpret from a semiotic perspective in order to explicate the nature and function of symbolic mediation with heightened emphasis.

Mead's notion of perspective is not built on a tension between subjectivity and objectivity. The fact that he argued for objective perspectives might be misleading if regarded from within a framework where personal perspectives are identified with subjective takes on the world and objectivity with an impersonal perspective, or a lack of perspectivity. An objective perspective is not an impersonal perspective. First of all, the Meadian notion of perspective was motivated by the need to overcome the cleavage between physicalism of the sciences that reduce reality ultimately to physical particles and trace any other object to the effects of these on consciousness, and any form of idealism that reduces reality to consciousness. A world that is conceived in a way that is independent of any organism would be without perspectives. In such a conception of the world that is independent of perspectives there cannot be any objects beyond physical particles, since any object beyond these is constituted through abstraction from real relations that are grounded in the attitude of an organism which maintains itself through its relation to the world (1972, p. 165).

On the other hand, the idealist take on consciousness divorces mind from the world to the effect that it became a most difficult task for posterity to place the mind in a world that was rendered completely alien to it. The task, for Mead, could only be attained if perspectivity is 
recognized as an ontological principle and the object is conceived in terms of the real relation between the organism and the environment; as the third term of this relation, whose existence depends ontologically on it: "the perspective is the world in its relationship to the individual and the individual in his relationship to the world" (1972, p.115).

The field of objects grounded in the dynamic, diachronic perspective of interactive conduct yields a particular environment that goes beyond an exclusive schism of subjectivity and objectivity. From the most fundamental organismic activity to the most complex reflective action, all conduct takes place necessarily within an environment whose reality consists in its relationality-an Umwelt, or a lifeworld. Conceived from a pragmatist point of view, aspects and elements of this relational environment emerge simultaneously with the agent through its meaningful conduct within interactive situations. Mead grounds the object-hood of things ultimately in the perspectivity of conduct, which implies, though, not a subjective givenness of the lifeworld as the background of conduct but the situatedness, and implicitly or explicitly reciprocal, or in Mead's terms social (1972, p. 189), orientation of unitary conduct. In a similar vein, Jack Martin articulates Mead's concept of perspective in terms of "holistic (perceptual, conceptual, emotional, esthetic, and physical) orientations to situations with a view to acting within them" (2008, p. 299).

Objects are neither passive, self-identical, or mono-dimensional with respect to conduct, nor can be reduced to the contents of a subjective consciousness. Conceived thus, object-hood is not restricted ontologically to any entity, nor is any entity entitled to object-hood prior to conduct. Since the object is grounded in the perspective of conduct, its reality is the feature of the nature and structure of the conduct and the particular relational environment within which it takes place. Thus, a piece of furniture is not an object in the relational environment of an insect, whose conduct does not involve such a differentiation that could bring a table to the foreground in its unity among other aspects of the environment, nor is a "person" with own beliefs and desires in that of a cat. Others come forward as friends, legal subjects, voters, organisms, or even solid bodies when approached with corresponding attitudes in corresponding situations and respond accordingly. One becomes an object in the social world to the extent that he or she participates in social situations embedded in the common world of social meanings. One becomes an object for oneself to the extent that one's conduct involves a reference to oneself as an integral part, which presupposes that others become objects featuring in the conduct and oneself comes to occupy a similar role. Mead puts this self-referencing in one's conduct as taking the attitude of the other on oneself, where the term attitude is equivalent to the term perspective, and the other in question is a social object that might denote a "specific other", such as the mother for the child, a friend, a partner; a societal role or identity, such as the speaker or the listener, a judge, a teacher; or a "general other" as standing for societal values, norms, rules and the like. One's "self," thus, can be as complex and multi-layered as "the other" and "the lifeworld" are. The lifeworld of the three-year-old does not have moral codes, institutions, laws, or scientific objects and others who are dignified, guilty, melancholic or opportunistic; but in difference to the lifeworlds of presumably many other animals it features jokes, imitation, false beliefs, role-taking, and pretend play, which require taking and coordinating different perspectives on social objects. 
Thus, perspectivity is a necessary feature of the meaning-making relation the individual enters with the world through action, and any organism with a relational environment can be said to have a perspective. The nature and semiotic complexity of the meaning-making relation to the environment depends on what aspects of the environment are rendered meaningful and what kind of response is given to those meanings. Such a meaning-making relation involves an internal system of values that mediate responses. A stone cannot have a perspective because there is no such mediation through internal values and no resulting "world" which would be associated with it. Jacob Uexküll conceived this mediation as the mark of the living, and argued for the presence of a perspective even without consciousness. Even the most "automatic" responses of simple organisms in an environment manifest meaning, although non-conscious. In contemporary semiotic terms, the simplest meaning-making relation to the world involves a filtering of environmental stimuli in terms of relevance, rendering or transduction of environmental cues into organismic signals, and measurement. Even the simplest meaning-making activity, thus, takes place from within a perspective. The peculiarly human meaning-making processes, though, require going beyond a singular perspective and assuming, changing, coordinating various perspectives within the unity of action. This qualitative difference between the perspectivity of human action and those of many other organisms should be sought in the difference between the operative sign processes. For our purposes, we can categorize signprocesses in three levels of complexity.

Signal-response: This level corresponds to the perspective of the simplest organisms. An example is found in Uexküll's famous description of the Umwelt of a tick, who climbs according to the sun light to the tip of a branch in non-conscious anticipation and hangs there until a specific odor emanating from the sweat of a mammal draws it to drop from there on top of the mammal. It then finds a hairless spot and feeds on the blood. Its Umwelt features specific shades of light, odors, heat, tactile qualities and not the sun, the trees, the animals, or blood that are featured in ours. There are no objects at this level. There is a direct connection between the signal and the corresponding action, and hence no differentiation between sign and object. The mammal is not indicated by the odor; it is the odor. Meanings, hence, are not explicit for the tick but implicit in its behavior. Its Umwelt cannot be articulated as a world. Consequently, it is immersed and entrapped within its perspective.

Sign-object relations: Complex animals have perspectives that are based on more than signal-response processes: there are sign-object relations which exploit similarity, difference, patterns, regularities and involve agentive interaction with the environment. Signs can be identified as distinct from their objects. There is a world of relational objects that can be responded to in different ways. Behavior is not immediate and reactive but mediated; there is affectivity and memory, hence expectation, surprise, desire, frustration. There are choices in the environment, or semiotic freedom, which presupposes that "future" is integrated into the system of internal values. Mead's analysis of the social act, which we will turn to in the subsequent section begins at this level where it is possible to talk about a communication of gestures: certain responses can become signs for others through being interpreted as indicating future phases of an interactive situation and thereby offering social action possibilities. 
Sign-sign relations: These are not only iconic and indexical but also symbolic in character. ${ }^{5}$ The internal system of values has reflexivity, a reference to itself as such. Symbol relations, or sign-sign relations can also be described in terms of meta-semiosis, where semiosis is understood as iconic and/or indexical relations. This type of higher order meaningmaking implies that the sign-relations are interpreted as signs themselves, which in turn enables that the meaning-making relation to the world can itself be the object of meaning-making activity. In other words, in symbols embodied, relational meaning can be articulated and become a sign itself. Perspectives can be articulated, hence can be made a constituent of the social act, be distanced from, changed, coordinated. The particular property of symbols that enable perspective-taking is, according to Vygotsky, reverse action or, according to Mead significance: they can be stimuli as well as response and elicit the same response in oneself as they do in the other. Just as we simultaneously listen to ourselves as we speak, the inherently perspectival nature of symbols also allows us to convey something to ourselves in the manner we do so to others.

Higher order signs, or symbols, come about only through a history of social interaction. They are ultimately conventional in the sense that they are not implicit features of natural acts but explicit features of social acts, but they are objective in the sense that they are embedded in a system of common socio-cultural meanings, that are grounded in but are not reducible to organismic meaning. Sign-sign relations not only make objective, shared meaning possible as Mead contends; but also, they free thinking and affectivity from the concrete constraints of here-and-now. In Vygotsky's terms they liberate meaning from the object and action from direct response, to the effect that the individual acts not under the domination of what the object immediately calls for but on social meanings. Tomasello (1999) maintains, in a similar vein, that the perspectival nature of symbols allows their users view and to induce other people to view the world in certain aspects rather than others or in a manner that surpasses the directly perceivable qualities and affordances. He argues that internalizing a symbol also amounts to understanding the human perspective embodied in it and opens up a novel dimension to the reach of human intentionality. Accordingly, acquiring a symbolic language allows children to distance themselves from the contents of their immediate experience and take a higher order perspective on their actions. One of the central aspects of language acquisition, for Tomasello, is developing a particular conceptual perspective on the world. Perner et al. (2002) refer to another aspect of the perspectival nature of symbols. They argue that different sortals applied to the same thing, such as Dalmatian, dog, and animal, individuate it from different truthcompatible conceptual perspectives, just as several perceptual perspectives on the same object yield different but compatible descriptions of it. Synonyms used in alternative naming tasks similarly require from the child to coordinate different, non-exclusive perspectives on a common object of reference. Using Frege's distinction, we can say that the ability for connecting different senses with the same reference, as in the case of synonyms, is at bottom an ability for perspective-coordination.

Interpersonal symbolic communication is characterized and determined by a common, yet multi-dimensional field of social meanings that have a general, societal, or group level, which

\footnotetext{
${ }^{5}$ Symbols, for Peirce, are general signs which refer to their objects independently of both the properties of the sign and of any actual relationship between the sign and the object.
} 
becomes the perspective of society or in Mead's terms the "generalized other" when symbolically articulated, and innumerous particular re-presentations articulated in the form of personal perspectives — once again in Mead's terms perspectives of "specific others" and "me." In this vein, interpersonal communication takes place between "me" who is engaged paralelly in autocommunication and a "person." A person is a symbolical other and in that an internal, represented other as much as an external, encountered other. Heterocommunication as well as autocommunication is structured by an infinity of symbol-relations which are formed outside of the particular interaction - be it social, cultural, historical, or moral - and place constraints that override the singularity of the hic et nunc and render the communicative situation possible as such; i.e., meaningful. The context of the systematically organized symbol relations within which the communicative situation is embedded renders it general and thus in principle repeatable, even though it would never be repeated in exact sameness. This does not mean, as it is self-evident, that persons are general terms and the conveyed meanings are of a universal nature; persons are particulars and meaning is always already local, historical, and contextdependent, since interrelations of symbols bring about an inexhaustably infinite horizon of meaning and the activity of interpretation continuously particularizes. Yet singularity cannot be symbolically represented and grasped. It always aludes the interpreting activity since it does not belong to the domain of the semantic but to that of the pragmatic, namely that of the act and of the event. Mead conceives this spontaneity of the act in terms of the notion of "I", which is the spontaneous and only retrospectively discernable response to the "me". To articulate in other words, if the perspectivalness of the social world and the unsaturatedness of the symbol is necessitated on the one hand by our interpreting relation to the world-that the universals making up the perspective of society always have to appear in the communicative situation in the particularized form of personal perspectives, it is so on the other by virtue of the spontaneity of the act and the singularity of the event.

\section{Pre-symbolic interaction as the ground of symbolic communication}

Both Mead and Vygotsky were concerned with grounding signs of culture in a way that would avoid explaining the origin of mental forms with yet other mental forms and the constitution of meaning on the basis of individual meaning making, which risks falling into circularity, an innatism with respect to intellectual powers, or solipsistic reduction with respect to meaning. Sign-using activity, which both Mead and Vygotsky see as the cradle of the reflective mind, must be grounded in something that is originally something else. Vygotsky (1978, p. 46) writes:

[...] sign-using activity in children is neither simply invented nor passed down by adults; rather it arises from something that is originally not a sign operation and becomes one only after a series of qualitative transformations. Each of these transformations provides the conditions for the next stage and is itself conditioned by the preceding one; thus, transformations are linked like stages of a single process, and are historical in nature [...] In the history of behavior these transitional systems lie between the biologically given and the culturally acquired. We refer to this process as the natural history of the sign.

In a similar fashion, for Mead thinking presupposes communication and not the other way around. And communication does not presuppose the existence of minds, which would render 
the origin of mind and the interaction among minds mysteries. If we take, on the other hand, social processes of experience and behavior in some rudimentary form to be primary and explain the origin of minds in terms of the interactions within a social context of experience, then neither the mind, nor the interaction among the minds seems mysterious (Mead, 1972, p. 50). In other words, identifying mind and subjectivity as first-order phenomena and explaining communication and intersubjectivity on their basis is putting the cart before the horse; these are rather just as real, but explanation-wise second-order phenomena.

Meaning must be construed from the start not as individual but as shared, or co-created meaning, and the powers for appropriating and comprehending it must be understood in terms of their development. In short, the essence of the sign process was to be looked for in the social history of the sign. The ground they both arrived at was non-symbolical communication, in gestures. In other terms, they both looked at mimesis for the evolutionary as well as developmental origins of symbolically mediated activity.

For Mead, gesture is the beginning of the social act, which is "that phase of the individual act to which adjustment takes place on the part of other individuals in the social process of behavior" (1934, p. 47). He gives the example of two dogs approaching each other with a hostile attitude as a case of conversation of gestures:

The act of each dog becomes the stimulus to the other dog for his response. There is then a relationship between these two; and as the act is responded to by the other dog, it, in turn, undergoes change. The very fact that the dog is ready to attack another becomes a stimulus to the other dog to change his own position or his own attitude. He has no sooner done this than the change of attitude in the second dog in turn causes the first dog to change his attitude. We have here a conversation of gestures. They are not, however, gestures in the sense that they are significant. We do not assume that the dog says to himself, "If the animal comes from this direction, he is going to spring at my throat and I will turn in such a way." What does take place is an actual change in his own position due to the direction of the approach of the other $\operatorname{dog}(1934$, p. 42-3).

The gesture indicates for the addressee, the resultant of the social act thereby initiated. The social act is meaningful, and the behavior of the first individual is a gesture, if this relation of indication holds between the three phases of the social act - the initial gesture, the response of the other individual to the first gesture, the resultant of the social act. The gesture, in the peculiar terminology of Mead, is in a sense the symbol of the resultant of the social act. The relation need not be consciously represented as such by the participants of the social act; the gesture need not be a significant symbol in order to initiate a social act, but any significant symbol is a gesture embedded in a social act. The gesture becomes significant when it influences the actor in the same way it influences the observer:

When [...] that gesture means this idea behind it and it arouses that idea in the other individual, then we have a significant symbol. In the case of the dog-fight we have a gesture which calls out appropriate response; in the present case we have a symbol which answers to a meaning in the experience of the first individual and which also calls out that meaning in the second individual. Where the gesture reaches that it has become what we call "language.” It is now a significant symbol and it signifies a certain meaning (1934, p. 46-7) 
Meaning can become explicit in the peculiarly human form of communication, and develops out of the matrix of social acts in terms of symbolization. Symbolization establishes the condition for reflexivity, hence of consciousness, by virtue of its giving rise to the same attitude towards a common object, calling out a common response, in all the participants of the social act. The snarling of the dog, in Mead's example, effects a change in the attitude of the second dog, but it does not effect this change in the first dog. In other words, it is a stimulus for the second dog, arousing a tendency to fight back or to flee, but not for the one who is snarling. In the terminology of Vygotsky, the gesture does not have the property of reverse action, that is, the property of being at the same time a stimulus and a response. If the gesture has this property, it can become a stimulus with the same meaning both for the other and for the self, and brings about the possibility of not only effecting change and adjustment in the attitude and behavior of the other but also in that of one's own. If the gesture communicates the same meaning to the individual showing it as it does to the other, he is entertaining two perspectives: besides his own organized attitude he is aware of the resulting attitude of the other, and this awareness already factors in his initial attitude. A hunting party arranges stones in a pile at a particular location, a response, and the pile becomes a stimulus for a second party to look for game in that area. To take an example from Vygotsky (1978, p. 127); an individual ties a knot on a handkerchief, a response, in order later to remember something through its mediation, a stimulus. But the social grooming gesture of a chimpanzee also fulfills this feature, although to a lesser degree, as arousing the same meaning in both parties.

As to how gestures come to acquire significance in the course of development, it is worth to turning to Vygotsky. One often quoted example of his from the pre-symbolic stage is the mimetic development of a most crucial gesture-pointing:

Initially, this gesture is nothing more than an unsuccessful attempt to grasp something. [...] The child attempts to grasp an object placed beyond reach; his hands, stretched toward that object, remain poised in the air. [...] At this initial stage pointing is represented by the child's movement, which seems to be pointing to an object-that and nothing more. When the mother comes to the child's aid and realizes his movement indicates something, the situation changes fundamentally. Pointing becomes a gesture for others. The child's unsuccessful attempt engenders a reaction not from the object he seeks but from another person. Consequently, the primary meaning of that unsuccessful grasping movement is established by others. Only later, when the child can link his unsuccessful grasping movement to the objective situation as a whole, does he begin to understand this movement as pointing (1978, p. 56)

While the initial grasping movement is a stimulus for the mother, who alone attributes it meaning, but only a response to the stimulus of a thing for the child, it later becomes a stimulus for the child as well. The child then can perform the gesture to control the behavior of others-for instance, to direct their attention, and still later he can perform it to control his own behaviorfor instance, to guide his gaze on the blackboard while learning to read. It might be said that the same is the case in the child's transition from crying as affective discharge, to crying as a gesture of calling the mother, in order to trigger an interaction that is anticipated, which in turn gives way to crying out the word "mommy". 
While Vygotsky's account of sign operation and Mead's account of the significant gesture is strikingly similar, their emphases are slightly different. While the major concern for Mead is the construction of social meaning and social selves, for Vygotsky it is mental development. The key point for Mead is that in significant communication the phases of the social act are organized in terms of roles, which can be exchanged, since the reversibility of the gesture enables commonality of meaning. This brings about reflectivity and hence opens individual behavior for control in accordance with a social system of meaning. For Vygotsky it is that the sign operation transforms the structure of psychological operations and thereby natural acts give way to mediated acts characterizing culture.

The question is how this development from non-symbolic, mimetic activity to symbolic activity takes place. Both for Mead and for Vygotsky, this is a process of internalization of an external process. But while for the former, the emphasis is on the internalization of social meaning through perspective taking and constant exchange of social roles in interactions, which ultimately results in the inner construction of a self and a generalized other, for the latter it is on the internalization of social and cultural forms of mediated activity through structural transformation of psychological operations.

\section{Symbolic activity and development}

The development of the capacity for symbolic activity occurs in various stages within a social context. It depends at any stage on the transformation of an intersubjectively realized activity into an intrasubjective one. Vygotsky explicates this process as "the internal reconstruction of an external operation" (1978, p. 56) and argues famously that:

every function in the child's cultural development appears twice, on two levels. First, on the social, and later on the psychological level; first, between people as an interpsychological category, and then inside the child, as an intrapsychological category (1978, p. 128)

The process of internalization that is explicated by Vygotsky is not a simple transmission of social relations, but comprises a series of structural transformations in the child's psychological operations. A paradigmatic case is the transformation of communicative speech to inner verbal thinking via egocentric speech, as the phenomenon was famously called by Piaget.

The phenomenon itself consists in audible verbal articulations of small children that mostly accompany their practical activity and are not produced in an interactive context or done so without any perceivable communicative intent. Through the course of development egocentric speech disappears roughly around the time children reach school age. This self-centered, noncommunicative speech displays certain structural differences from communicative, social speech. It does not follow the common grammatical sequence of a language to the completion of full sentences, it is mostly abbreviated full of omissions, and it cannot be understood by others if it is divorced from the concrete situation within which it takes place-for example through being written down or recorded. Piaget's opinion was that egocentric speech simply accompanied the activity of the child without having any significant bearing on it. He concluded from his observations that egocentric speech did not serve any necessary or useful function; it did not ameliorate in any concrete way the child's problem-solving ability and was 
not otherwise efficacious in shaping his experience. As well as not having any effect, the fate of egocentric speech was presumed by Piaget to be atrophy; to simply disappear by the time the child reaches school age.

Piaget's metapsychology, according to Vygotsky, in effect prevented him from seeing a formative connection between sign use and practical activity. Although Piaget was a pioneer in tracing the origins of concept formation to action, he could not associate the origins of the communicative function of the sign with the origins of its representative function. In other words, he could not see a constitutive relation between communication and thinking.

Besides adopting a stance that one can clearly associate with pragmatism, Vygotsky evaluates his empirical and experimental findings on egocentric speech within a theory on the nature and development of verbal thinking. According to Vygotsky, egocentric speech constitutes a paradigmatic case of how sign use, instead of being subsequently added to the independently developed structures of practical activity, becomes a constitutive factor in the development of higher psychological functions through being intertwined with practical activity, and how the course of human ontogeny follows a structurally novel route by being embedded in a culturally mediated environment:

Instead of appealing to the adult, children appeal to themselves; language thus takes on an intrapersonal function in addition to its interpersonal use. When children develop a method of behavior for guiding themselves that had previously been used in relation to another person, when they organize their own activities according to a social form of behavior, they succeed in applying a social attitude to themselves. The history of the process of the internalization of social speech is also the history of the socialization of children's practical intellect (1978, p. 27)

Egocentric speech is not a first-order phenomenon, but an intermediary one. It derives from social communication, which is the first-order phenomenon, and through structural transformations gives way to reflective, verbal thinking. It is a transitional phenomenon which is externally similar to communicative speech, yet its function is not communication. Egocentric speech is the functional basis for inner speech, but in its external form it is embedded not in inner speech but in communicative speech $(1978$, p. 28). What takes place during this transitional phase is actually an attempt on the child's part to recreate the situation of practical activity under the guidance of a parent or instructor in the form of a solitary activity that is accompanied and later guided by the child's egocentric speech. As the child's attention is guided by another in the interactive situation by naming and pointing to objects, the child points to and calls out the names of objects in order to direct his attention and physical approach. They begin to ask themselves questions in the face of confusion, hardship or failure that are similar to the ones posed to them before by a competent other, but without formulating them in clear grammatical structures and without awaiting any answer. In later stages, the child begins to articulate possible actions, in a similar fashion to the suggestions given by another in the interactive situation, before engaging in them.

The function of egocentric speech lies in guiding the child's attention and behavior, in enabling more efficient manipulation of objects through freeing the child's activity from the constraints of the immediate visual field, and eventually in changing the temporal structure of the child's experience through beginning to use language for planning actions: "children solve practical 
tasks with the help of their [egocentric] speech, as well as with their eyes and hands. This unity of perception, speech, and action, which ultimately produces internalization of the visual field" is at the core of peculiarly human forms of behavior $(1978$, p. 26). At the beginning, speech follows the child's actions and is dominated by activity. Later, speech begins to occur at earlier phases of an activity and is eventually moved to the starting phase, which marks the emergence of a new relation between word and action: "Now speech guides, determines, and dominates the course of action; the planning function of speech comes into being" (1978, p. 28). As a result, a view of the future becomes an integral part of the child's physical movement in space and guides his approach to elements of his surroundings. He no longer acts under the constraints of the immediately given in the visual field, and is able to ignore the direct line between the act and the goal. All in all, with the help of this internally directed external speech the child becomes able to become the object of his own action as well as its subject.

Development, for Vygotsky as well as for Mead, follows a path from performance to competence with respect to social meaning. The entry gate into the social world for the child is mimetic activity, which depends initially heavily on imitation. Children need to master the forms of acts before mastering their meanings. This is also evident in that they appropriate reference before conceptual meaning. The child needs to observe and imitate other's behavior of referring in numerous social contexts, under constant guidance and correction by others, before he can conceptually represent the social object. The same is the case with using tools, applying learning strategies, adjusting to etiquette, holding one's body appropriately, or keeping a socially acceptable personal space.

For Mead, mastering social meanings takes place within institutional structures comprising of reversible social roles characterizing human societies (Gillespie, 2005). As stated in the preceding, comprehension of meaning depends on taking on the attitude of others towards one's own action. This activity requires the ability to coordinate different perspectives on a common social object, the simplest case of which is the coordination of actor and observer perspectives. The significant symbol, especially the vocal gesture, enables this by virtue of affecting the actor and the observer in the same manner. It can be articulated and heard, written and read, enacted and observed. Yet, mastering the significant symbol requires the internalization of the whole structure of communication in order for it to enable taking and coordinating different perspectives. This is not a straightforward process. The child can come to take different perspectives, and thereby to represent other attitudes, only through actually occupying all the roles indicated in a social process. The peculiar institutional structure of social processes in human societies is a necessary condition for this, where not only differentiation of roles such as fighters, resource gatherers, nest builders are featured but also collective coordinated activity and frequent position exchange. The majority of social acts in human societies involve reversible positions: giving and taking, talking and listening, calling and coming, mourning and consoling, helping and receiving help, leading and following, buying and selling, teaching and learning, punishing and receiving punishment, or winning and losing. From birth, children begin to engage in taking a role in such social acts. They engage in coordination of attention and timing of vocalizations as babies, as toddlers they play throw and catch, or hide and seek. Later, they experiment in social roles during pretend play; they become doctors and patients, parents and children, hosts and guests, and still later they can adjust themselves to the rule based structure of social games. Through performing in different positions in social acts, children come to 
experience complementary perspectives. They become able in time to represent the complex perspectival structure of social acts. Representation, as argued before, is grounded not in a mental but practical, enacted experience of perspectives. But it cannot be realized without engaging in significant communication, that is, without some form of language.

The origin of the self also lies, for Mead, in the process of assuming the other's perspective on one's action. The individual organism comes to become a self through the other. This is not the case in the case of the conversation of gestures, very complex forms of which are found in non-human animals, since "the conversation of gestures [...] does not carry with it the reference of the individual, the animal, the organism, to itself" (1934, p. 145). Only significant symbols involve this, and only through a history of significant communication can selves arise. The self, hence, is not presupposed by Mead in logical, experiential, or biological terms for the existence of sociality or symbolical communication. He writes (1934, p. 195):

When a self does appear it always involves an experience of another; there could not be an experience of a self simply by itself. The plant or the lower animal reacts to its environment, but there is no experience of a self [...] When the response of the other becomes an essential part in the experience or conduct of the individual; when taking the attitude of the other becomes an essential part in his behavior - then the individual appears in his own experience as a self; and until this happens he does not appear as a self.

The self is a social process, whose possibility lies in its being a part of the much wider, social and historical process. The primal objectification of the self is, thus, the process of taking the attitude of the other. But this yields a momentary and transient selfhood. The self acquires an enduring, yet dynamic, identity through time and across social interactions by internalizing a generalized other, which represents the whole of the social process. The generalized other is a symbolized unity that comprises the rule-based organization of social roles. It develops out of a history of social interactions with "specific others," where the participants increasingly focus on the rules governing the above mentioned institutional structures. The transition from play to games during the course of development is the transition from perspective taking to rule following, and marks the beginnings of the passage from taking the attitude of the specific other to taking the organized and generalized attitude of the generalized other-the society. In some form the whole social organization comes to be present in the consciousness.

In a similar manner, which also resonates with theories of executive functioning (e.g., Perner et al., 1999), Vygotsky describes play as where the child practices to control his impulses and control his behavior in a way which liberates it from the immediate situation:

in play, things lose their determining force. The child sees one thing but acts differently in relation to what he sees. Thus, a condition is reached in which the child begins to act independently of what he sees (1978, p. 96-7)

The first function of play is to sever the direct link between perception and meaning. Socialization to social meanings, competence therein, is dependent on transforming psychological operations from the situation where meaning is dominated by action and perception to that where action and perception are dominated by meaning. The evolution of children's play from pretense scenarios of role taking to games of rule following manifests a parallel change: 
The simplest game with rules [is also] an imaginary situation in the sense that as soon as the game is regulated by certain rules, a number of possibilities for action are ruled out [...] The development from games with an overt imaginary situation and covert rules to games with overt rules and a covert imaginary situation outlines the evolution of children's play (1978, p. 95)

As to the constitution of a self, Vygotsky states in a more explicitly dialectical wording, that the child "becomes for himself what he is in himself through what he manifests for others" (1978, p. 105). The structure of personality is constituted by internalized social relationships: the genetic structure, functioning, organization of all higher processes, their very nature is social. Even as purely psychological processes, they remain quasi-social.

Consequently, for both thinkers the development of a reflective mind and the constitution of an enduring yet dynamic self is a process of socialization and enculturation that takes its start as a mimetic participation in social situations, goes through internalization of external social activities mediated by the use of socially, culturally, and historically constituted symbols, and culminates in self-conscious competence of a social agent who is able to act upon generalized social meanings, as well as to negotiate them.

\section{Conclusion}

We have argued that in approaching social cognition, it is a worthwhile effort to go from interaction to thought and from social coordination of perspectives to meta-representational perspective-taking. The most suitable method for such a strategy is to place the emphasis on how social interaction brings about and is in turn deepened and enriched by symbolic activity. The social-relational tradition exemplified in the work of Mead and Vygotsky has the advantage of offering a holistic, historical and developmental perspective that does not dichotomize experience and cognition, interaction and representation, individual and social activity. By approaching perspectivity, not along dualistic themes, but on the basis of meaning-making activity within a relational environment, we can re-construe the activity of perspective-taking as social and situated as well as placing it at the center of all higher intellectual activity. The key to such an approach is to illuminate how sign-relations are grounded in action within the environment and how they acquire qualitatively novel forms through social interaction.

\section{References}

Astington, J., Harris, P. L., Olson, D. R.(Eds.). (1988). Developing Theories of Mind. Cambridge University Press.

Baldwin, J.M. (1906/ 1894). Mental development in the child and the race: Methods and processes (3rd rev. ed.). New York: Macmillan

Bartsch, K., \& Wellman, H.M. (1995). Children talk about the mind. New York: Oxford University Press.

Baron-Cohen, S., \& Ring, H. (1994). A model of the mindreading system: Neuropsychological and neurobiological perspectives. In C. Lewis \& P. Mitchell (Eds.), Children's early understanding 
of mind: Origins and development (pp. 183-207). Hillsdale: Lawrence Erlbaum Associates

Blumer, H. (1969). Symbolic interactionism: Perspective and method. Englewood Cliffs, NJ: Prentice-Hall.

Bruner, J. S. (1990). Acts of meaning (Vol. 3). Cambridge: Harvard University Press.

Carpendale, J., \& Lewis, C. (2006). How children develop social understanding. Oxford: Blackwell.

Carruthers, P. (2009). How we know our own minds: The relationship between mindreading and metacognition. Behavioral and Brain Sciences, 32(2), 121-38.

Clark, E. V. (1997). Conceptual perspective and lexical choice in acquisition. Cognition, 64 (1), 137.

Cole, M. (1985). The zone of proximal development: where culture and cognition create each other. in J.V. Wertsch (ed.), Culture, Communication and Cognition: Vygotskian Perspectives (pp. 146-161). Cambridge: Cambridge University Press.

Cole, M., Engeström, Y., Vasquez, O. (1997). Mind, culture and activity. Cambridge: Cambridge University Press.

Côté, J-F. (2016). George Herbert Mead's concept of society: A critical reconstruction. NY: Routledge.

De Jaegher, H., \& Di Paolo, E. (2007). Participatory sense- making: An enactive approach to social cognition. Phenomenology and the Cognitive Sciences, 6(4), 485-507.

De Jaegher, H., Di Paolo, E., \& Gallagher, S. (2010). Can Social Interaction Constitute Social Cognition? Trends in Cognitive Sciences, 14(10), 441-447.

De Jaegher, H. \& Froese, T. (2009). On the role of social interaction in individual agency. Adaptive Behavior, 17 (5), 444-460.

Edwards, A. (2007). An interesting resemblance. In H. Daniels, M. Cole, \& J. V. Wertsch (Eds.), The Cambridge companion to Vygotsky (pp. 77-100). Cambridge: Cambridge University Press.

Flavell, J. H. (2000). Development of children's knowledge about the mental world. International Journal of Behavioral Development, 24(1), 15-23.

Flavell, J. H., \& Miller, P. H. (1998). Social cognition. In D. Kuhn \& R. S. Siegler (Eds.), Handbook of child psychology: Vol. 2. Cognition, perception, and language (5th ed.), (pp. 851-898). New York: Wiley

Fuchs, T., \& De Jaegher, H. (2009). Enactive intersubjectivity: Participatory sense-making and mutual incorporation. Phenomenology and the Cognitive Sciences, 8(4), 465-486.

Gallagher, S. (2001). The practice of mind: Theory, simulation, or primary interaction? Journal of Consciousness Studies, 8 (5-7), 83-108.

Gallagher, S. (2008). Direct perception in the intersubjective context. Consciousness and Cognition, 17(2), 535-543.

Gallagher, S. \& Hutto, D. (2008). Understanding others through Primary Interaction and

Narrative Practice. In J. Zlatev, T. Racine, C. Sinha, \& E. Itkonen (Eds.), The Shared Mind: Perspectives on Intersubjectivity (pp. 17-38). Amsterdam: John Benjamins. 
Gallese, V., \& Goldman, A. (1998). Mirror neurons and the simulation theory of mindreading. Trends in Cognitive Sciences, 2(12), 493-501.

Gallese, V. (2014). Bodily selves in relation: embodied simulation as second-person perspective on intersubjectivity. Philosophical Transactions of the Royal Society B: Biological Sciences, 369(1644).

Gillespie, A. (2005). G.H. Mead: Theorist of the social act. Journal for the Theory of Social Behaviour, 35(1), 19-39.

Goldman, A. I. (2006). Simulating minds: The philosophy, psychology, and neuroscience of mindreading. Oxford: Oxford University Press.

Gopnik, A., \& Meltzoff, A. N. (1997). Words, thoughts, and theories. Cambridge, MA: MIT Press.

Gordon, R. M. (1986). Folk psychology as simulation. Mind and Language, 1(2), 158-171.

Martin, J., Sokol, B. W., \& Elfers, T. (2008). Taking and coordinating perspectives: From prereflective interactivity, through reflective intersubjectivity, to metareflective sociality. Human Development, 51(5-6), 294-317.

Mead, G. H. (1934). Mind, self and society. Chicago: University of Chicago Press.

Mead, G. H. (1972). The philosophy of the act. London: University of Chicago Press.

Mounoud, P. (1996). Perspective taking and belief attribution: From Piaget's theory to children's theory of mind. Swiss Journal of Psychology, 55(2/3), 93-103.

Nelson, K. (1998). Language in cognitive development: The emergence of the mediated mind. Cambridge University Press.

Peirce, C. S. (1931-58). The collected papers of Charles Sanders Peirce (Vol. 1-6 edited by C. Hartshorne \& P. Weiss, volumes $7 \& 8$ edited by A. W. Burks). Cambridge, MA: Belknap Press of Harvard University Press (cited as CP).

Peirce, C. S. (1992). The essential Peirce: selected philosophical writings (Vol. 2). Bloomington, IN: Indiana University Press (cited as EP).

Perner, J. (1991). Understanding the representational mind. Cambridge, MA: MIT Press.

Perner, J., Stummer, S., \& Lang, B. (1999). Executive functions and theory of mind: Cognitive complexity or functional dependence? In P. D. Zelazo, J. W. Astington, \& D. R. Olson (Eds.), Developing theories of intention: Social understanding and self-control (pp. 133-152). Mahwah, NJ: Lawrence Erlbaum.

Perner, J., Stummer, S., Sprung, M., \& Doherty, M. (2002). Theory of mind finds its Piagetian perspective: Why alternative naming comes with understanding belief. Cognitive Development, $17(3-4), 1451-1472$.

Piaget, J. (1928). Judgment and reasoning of the child. New York: Harcourt Brace Jovanovich.

Piaget, J. (1962/1945). Play, Dreams and Imitation in Childhood. New York: Norton.

Piaget, J. (1977/1974). The grasp of consciousness: Action and concept in the young child. London: Routledge \& Kegan Paul.

Piaget, J., \& Inhelder, B. (1963/1948). The child's conception of space. London: Routledge \& 


\section{Kegan.}

Reddy, V., \& Morris, P. (2004). Participants don't need theories: Knowing minds in engagement. Theory \& Psychology, 14(5), 647-665.

Rogoff, B. (1999). Cognitive development through social interaction: Vygotsky and Piaget. In P. Murphy (Ed.), Learners, Learning and Assessment (pp. 69-82). London: Paul Chapman Publishing.

Rogoff, B. (2003). The Cultural Nature of Human Development. New York, NY, US: Oxford University Press.

Scholl, B. J. \& Leslie, A. M. (1999). Modularity, development and 'theory of mind'. Mind \& Language. 14(1), 131-153.

Shanton, K., \& Goldman, A. (2010). Simulation theory. Wiley Interdisciplinary Reviews: Cognitive Science, 1(4), 527-538.

Shantz, C. U. (1983). Social cognition. In J. H. Flavell \& E. M. Markman (Eds.), Handbook of child psychology: Cognitive development (pp. 495-555). New York: Wiley.

Varela, F., Thompson, E., \& Rosch, E. (1991). The Embodied Mind: Cognitive Science and Human Experience. Cambridge, MA: MIT Press.

Vygotsky, L. (1978/1930). Mind in society: The development of higher psychological processes. Cambridge, MA: Harvard University Press.

Vygotsky, L. (1987/1934). Thinking and speech. In R. W. Rieber \& A. S. Carton (Eds.), The Collected Works of L. S. Vygotsky. Vol 1: Problems of General Psychology. New York: Plenum.

Wertsch, J. V. (1998). Mind as Action. Cambridge, MA: Oxford University Press.

The editorial and publishing process of this publication has been financed by the Ministry of Science and Higher Education from the funds for the dissemination of research (DUN) within the framework of publishing activity, contract no. 711/P-DUN/2019, period of implementation: the years 20192020. 